\title{
Développement d'un programme innovant pour mieux soutenir les familles vivant avec un enfant présentant une déficience intellectuelle ou un trouble du spectre de l'autisme : s'inspirer des expériences des milieux communautaires
}

\author{
Élise Milot, Marie Grandisson, Anne-Sophie Allaire, Charlène Bédard, Martin \\ Caouette, Myriam Chrétien-Vincent, Justine Marcotte, Sébastien Moisan et \\ Sylvie Tétreault
}

Volume 64, numéro 1, 2018

URI : https://id.erudit.org/iderudit/1055890ar

DOI : https://doi.org/10.7202/1055890ar

Aller au sommaire du numéro

Éditeur(s)

École de service social de l’Université Laval

ISSN

1708-1734 (numérique)

Découvrir la revue

Citer cet article

Milot, É., Grandisson, M., Allaire, A.-S., Bédard, C., Caouette, M., Chrétien-Vincent, M., Marcotte, J., Moisan, S. \& Tétreault, S. (2018).

Développement d'un programme innovant pour mieux soutenir les familles vivant avec un enfant présentant une déficience intellectuelle ou un trouble du spectre de l'autisme : s'inspirer des expériences des milieux communautaires.

Service social, 64(1), 47-64. https://doi.org/10.7202/1055890ar

\section{Résumé de l'article}

$\mathrm{Au}$ Québec, les besoins de répit et de soutien des familles ayant un enfant présentant une déficience intellectuelle (DI) ou un trouble du spectre de l'autisme (TSA) sont nombreux et persistent. C'est ce qui a motivé un collectif de recherche composé d'acteurs de la ville de Québec à développer OASIS+, une offre de services de soutien à domicile et dans la communauté misant sur l'engagement et la formation d'étudiants universitaires issus des programmes d'ergothérapie et de travail social. Afin de guider le développement d'OASIS+ des entretiens ont été menés avec des représentants d'organismes communautaires de la province de Québec qui ont contribué à élaborer de tels services de soutien. Ils ont permis d'identifier des facilitateurs pour l'implantation d'une offre de services aux familles qui soit pérenne. Cet article présente les résultats de l'analyse de contenu thématique de ces entretiens. Miser sur l'engagement d'étudiants formés semble une avenue pertinente pour favoriser le mieux-être des familles.
Tous droits réservés (C) Service social,
Ce document est protégé par la loi sur le droit d'auteur. L'utilisation des services d'Érudit (y compris la reproduction) est assujettie à sa politique d'utilisation que vous pouvez consulter en ligne. 


\section{Développement d'un programme innovant pour mieux soutenir les familles vivant avec un enfant présentant une déficience intellectuelle ou un trouble du spectre de l'autisme : s'inspirer des expériences des milieux communautaires}

MILOT, Élise, Ph.D., professeure à l'École de travail social et de criminologie, Université Laval
et chercheure régulière au Centre interdisciplinaire de recherche en réadaptation et intégration sociale (CIRRIS)

GRANDISSON, Marie, Ph.D., professeure au département de réadaptation, Université Laval et chercheure régulière au CIRRIS

ALLAIRE, Anne-Sophie, M.A., professionnelle de recherche, CIRRIS BÉDARD, Charlène, étudiante à la maîtrise en travail social, Université Laval CAOUETTE, Martin, Ph.D., professeur au département de psychoéducation Université du Québec à Trois-Rivières et chercheur régulier au CIRRIS CHRÉTIEN-VINCENT, Myriam, étudiante au doctorat en sciences cliniques et biomédicales, Université Laval

MARCOTTE, Justine, étudiante à la maîtrise en santé publique, Université Laval MOISAN, Sébastien, étudiant à la maîtrise en travail social, Université Laval TÉTREAUlT, Sylvie, Ph.D., professeure, Haute École de travail social et de la santé de Lausanne (Suisse), Filière Ergothérapie

\section{RÉSUMÉ}

Au Québec, les besoins de répit et de soutien des familles ayant un enfant présentant une déficience intellectuelle (DI) ou un trouble du spectre de l'autisme (TSA) sont nombreux et persistent. C'est ce qui a motivé un collectif de recherche composé d'acteurs de la ville de Québec à développer OASIS+, une offre de services de soutien à domicile et dans la communauté misant sur l'engagement et la formation d'étudiants universitaires issus des programmes d'ergothérapie et de travail social. Afin de guider le développement d'OASIS+, des entretiens ont été menés avec des représentants d'organismes communautaires de la province de Québec qui ont contribué à élaborer de tels services de soutien. Ils ont permis d'identifier des facilitateurs pour l'implantation d'une offre de services aux familles qui soit pérenne. Cet article présente les résultats de l'analyse de contenu thématique de ces entretiens. Miser sur l'engagement d'étudiants formés semble une avenue pertinente pour favoriser le mieux-être des familles.

Mots-clés : familles, répit, soutien déficience intellectuelle, trouble du spectre de l'autisme. 


\begin{abstract}
In Quebec, respite needs of families with a child with intellectual disabilities (ID) or autism spectrum disorder (ASD) remain numerous and persistent. With this in mind, a research committee in Quebec City developed OASIS+, a program that offers a range of support services at home or in the community and that relies on the commitment of trained occupational therapy and social work university students. The aim of this paper is to present data from phone interviews with representatives of community organizations of different regions of the province of Quebec (Canada) who have contributed to the elaboration of this kind of services. The results, derived from a thematic content analysis, helped to guide the development of OASIS + . In conclusion, it seems that the involvement of trained students represents an effective strategy for fostering the well-being of families.
\end{abstract}

Keywords: families, respite, support, intellectual disability, autism spectrum disorder. 


\section{INTRODUCTION}

Au Québec, environ $2 \%$ des familles vivent avec un enfant présentant une déficience intellectuelle (DI) ou un trouble du spectre de l'autisme (TSA) (Camirand et al., 2010 ; Institut national de santé publique du Québec, 2017). II semble pertinent de définir rapidement ces conditions. La DI se manifeste par différentes limitations du fonctionnement intellectuel et du comportement adaptatif qui entravent de façon importante le quotidien des personnes (American Psychiatric Association [APA], 2013). Cet état apparaît avant l'âge de 18 ans. En ce qui a trait au TSA, il s'agit d'un trouble neurodéveloppemental caractérisé par des difficultés dans deux domaines : 1) la communication et les interactions sociales, et 2) les comportements, activités et intérêts restreints ou répétitifs (APA, 2013). Le niveau de soutien que nécessite la personne pour fonctionner dans sa communauté peut être situé sur un continuum allant du niveau I (nécessite un soutien) au niveau III (nécessite un soutien important). Plusieurs études montrent que ces conditions surviennent aussi souvent avec des comorbidités associées. Ainsi, une incidence plus élevée de problèmes de santé mentale et physique (p. ex. : épilepsie, diabète, troubles de l'appareil digestif) et de troubles de comportement est dénotée comparativement à la population générale (Brisson, Bolduc et Jourdan-Ionescu, 2014 ; Institut national de santé publique du Québec, 2017 ; Wallander, Dekker et Koot, 2006). Ces personnes sont aussi plus à risque de présenter des troubles moteurs, sensoriels ou langagiers (Jancarik, 2010 ; Munir, Friedman et Szymanski, 2015). Pour les parents, vivre avec un enfant, un adolescent ou un adulte qui présente une DI ou un TSA, ainsi que les comorbidités pouvant être associées à ces conditions, vient accroître les exigences en soutien et soins à prodiguer. D'ailleurs plusieurs études mettent en lumière que les parents vivant avec un enfant qui présente une DI ou un TSA expérimentent un niveau de stress élevé et sont particulièrement à risque d'épuisement et de détresse psychologique (Cappe et al., 2018 ; Ludlow, Skelly et Rohleder, 2012 ; Protecteur du citoyen, 2012). À ce sujet, une recension des écrits réalisée par Courcy et Des Rivières-Pigeon (2013) met en exergue que jusqu'à $50 \%$ des mères d'enfants présentant un TSA vivraient avec un niveau de détresse élevé. La perception de surcharge parentale vécue par les parents vivant avec un enfant présentant une DI ou un TSA peut avoir d'importantes répercussions sur leur santé physique et mentale, sur leur relation de couple et sur la conciliation travail-famille (Brannan et Heflinger, 2006 ; Feldman et al., 2007 ; Murphy et al., 2007 ; Ones et al., 2005 ; Woodgate, Ateah et Secco, 2008). De plus, le fait d'avoir un enfant ayant une DI ou un TSA peut entraver la réalisation de leurs activités domestiques quotidiennes (Myers, Mackintosh et Goin-Kochel, 2009) et leur participation à des activités sociales et récréatives (Bindels-De Heus et al., 2013 ; Courcy et Des Rivières-Pigeon, 2013 ; Luther, Canham et Young Cureton, 2005 ; Protecteur du citoyen, 2012). Parfois, des parents qui assurent la supervision constante de leur enfant ayant une DI ou un TSA rapportent se sentir coupables de ne pas avoir suffisamment de temps pour s'occuper de leurs autres enfants ou pour réaliser des activités familiales (Myers et al., 2009 ; Resch et al., 2010). Des chercheurs constatent aussi que plusieurs parents se sentent incompris par les membres de leur réseau social informel, lesquels remettraient en question leur manière de gérer les comportements de leur enfant (Ludlow et al., 2012 ; Siklos et Kerns, 2006). Certains perdraient aussi contact avec des personnes significatives, puisqu'ils doivent constamment décliner les activités qui leur sont proposées (Myers et al., 2009). Puisqu'ils déploient constamment des efforts d'adaptation et d'ajustement, les parents avec un enfant présentant une DI ou un TSA ont besoin de soutiens diversifiés pour être en mesure de se ressourcer et de concilier leurs rôles familiaux, sociaux et professionnels sans s'épuiser. 


\section{Contexte}

Au Québec, ces parents peuvent bénéficier de prestations et de subventions provenant des gouvernements provincial et fédéral pour avoir accès à des services de soutien et de répit. Les parents peuvent obtenir une compensation financière du Programme provincial de répit aux proches aidants pour recevoir des services de gardiennage, répit et dépannage. Cette aide vise à compenser la charge considérée " anormale " de leurs responsabilités parentales et à leur permettre de s'offrir un moment de détente et de ressourcement, souvent essentiel à la poursuite de leurs obligations (Vézina, Bourdages et Talbot, 2015). Selon ces derniers auteurs, cette compensation contribuerait, indirectement, à prévenir ou à retarder le placement des enfants. II faut préciser que la somme allouée à chaque famille est déterminée au moyen d'une évaluation de leurs besoins par un intervenant du Centre intégré (universitaire) de santé et de services sociaux (CISSS ou CIUSSS) ${ }^{1}$ de leur région. Le montant alloué est directement relié aux limitations de la personne à charge présentant une DI ou un TSA, à son âge, aux besoins de la famille et au montant de la subvention annuelle maximale établie par le $\mathrm{Cl}(\mathrm{U}) S S S$. Or, les sommes disponibles pour ces allocations aux familles québécoises varient en fonction du budget, qui fluctue grandement d'une région à l'autre en raison de décisions administratives différentes. De plus, les conditions d'accès à ces allocations sont souvent assez restrictives (p. ex. : interdiction d'embaucher un membre de la famille, imposition d'un âge d'admission).

Malgré l'offre de telles compensations financières, l'obtention de services adaptés est parsemée d'obstacles dans un contexte de ressources humaines limitées, de contraintes budgétaires et de besoins persistants. À titre d'exemple, les parents qui souhaiteraient bénéficier des services de répit des ressources communautaires se heurtent bien souvent à de longues listes d'attente, à des disponibilités limitées et parfois, à des critères spécifiques qui réduisent l'accès à certaines familles (Conseil de la famille et de l'enfance [CFE], 2008 ; Poirier et Des Rivières-Pigeon, 2013 ; Protecteur du citoyen, 2009). D'autre part, certains parents préfèrent se priver de tels services parce qu'ils estiment que le personnel est peu outillé pour composer avec les particularités de leur enfant ou que les conditions en ces lieux (mobilité du personnel, variété des atteintes des enfants) suscitent trop d'anxiété chez leur enfant (CFE, 2008). Dans son avis, le Conseil de la famille et de l'enfance (CFE, 2008) constate que ce sont souvent les services de répit qui déterminent le moment où l'enfant peut être accueilli, et ce, à des dates fixées à l'avance pour l'année. À ce propos, plusieurs parents dénoncent le manque de flexibilité de ces services et la difficulté à les utiliser selon leurs besoins réels ou lors de situations d'urgence. Des services de répit sont aussi offerts dans des ressources privées, mais les coûts en sont très élevés. À l'été 2016, une consultation régionale menée auprès de parents de Québec ayant un enfant présentant une DI ou un TSA ${ }^{2}$ mettait en lumière un besoin permanent, non comblé et partagé par la majorité des parents, soit celui de pouvoir accéder à une personne-ressource de confiance, en mesure d'intervenir adéquatement auprès de leur enfant et disponible pour leur offrir du

\footnotetext{
1 Dans la province de Québec, il y a 13 CISSS et 9 CIUSSS qui sont des mégastructures organisationnelles publiques créées à la suite d'une importante fusion d'établissements de santé et services sociaux. Ils constituent des points de référence régionaux où la population peut s'adresser en cas de problèmes psychosociaux ou de santé. La population peut y recevoir les services appropriés ou être dirigée vers une autre ressource de sa région d'appartenance. Pour plus d'informations sur le sujet, consultez ce lien : http://www.msss.gouv.qc.ca/reseau/reorganisation/portrait.

2 Cette consultation a été réalisée à l'été 2016, à partir d'un sondage soumis aux parents membres de l'Association pour l'intégration sociale de Québec ou d'Autisme Québec, deux organismes communautaires impliqués auprès des familles d'enfants ayant une DI ou un TSA.
} 
répit à domicile. De telles ressources sont rares et leurs services sont onéreux (CFE, 2008). Encourager les parents à assumer de tels frais pourrait alimenter les inégalités sociales entre les familles.

\section{OASIS+ : un programme innovant}

Miser sur le développement de stratégies innovantes pourrait permettre à ces familles d'accéder à des ressources répondant à leurs besoins réels en répit et en soutien à la participation sociale. C'est dans cette optique qu'un collectif de recherche composé de différents acteurs ${ }^{3}$ de la ville de Québec a développé OASIS+, une offre de services de soutien à domicile et dans la communauté misant sur l'engagement d'étudiants universitaires issus des programmes d'ergothérapie et de travail social et formés à cette fin. Cette stratégie s'inspire d'une des neuf recommandations formulées dans le cadre d'une vaste étude québécoise menée auprès de 200 acteurs dans le domaine (p. ex. : intervenants, familles, décideurs politiques) (Tétreault et al., 2012a, 2012b). Elle apparaît prometteuse, puisque de nouvelles cohortes d'étudiants sont accueillies chaque année, ce qui pourrait contribuer à la pérennisation du programme. De plus, tous les étudiants n'ont pas la possibilité de vivre des expériences avec les familles qu'ils seront appelés à côtoyer comme professionnels. Pourtant, de telles expériences peuvent contribuer au développement de savoir-agir fort pertinents à l'exercice de leur profession.

Pour s'assurer qu'OASIS+ soit un programme durable qui réponde aux besoins des familles et qui favorise l'implication active et le développement des compétences nécessaires chez les étudiants, une collecte de données a été réalisée en trois temps ${ }^{4}$.

Temps 1. Pour choisir des termes adéquats et bien définir les différents types de soutien à offrir aux familles à domicile et dans la communauté, l'équipe de recherche a recensé des écrits scientifiques et documents gouvernementaux pertinents sur le sujet (Agence de la santé et des services sociaux de la Capitale-Nationale, 2009a; Agence de la santé et des services sociaux de la Capitale-Nationale, 2009b ; Office des personnes handicapées du Québec, 2009 ; Tétreault et al., 2012a ; Tétreault et al., 2014). Sur cette base, trois principaux types de soutien ont été identifiés et validés par les membres du collectif de recherche. Ceux-ci sont: (a) l'accompagnement de l'enfant ou de la famille dans des activités sociales, sportives ou de loisirs ; (b) l'assistance aux parents dans la réalisation des tâches liées à la routine familiale ; (c) le gardiennage de l'enfant ayant des besoins particuliers (aussi nommé "présence-surveillance » lorsqu'il s'agit d'un adulte), avec ou sans la fratrie, lorsque le ou les parents s'absentent du domicile. Le gardiennage peut s'effectuer pour une période de courte ou de longue durée (quelques heures à quelques jours). II peut aussi s'actualiser de manière récurrente ou occasionnelle (p. ex. : dépannage lors d'imprévus). Temps 2. Des parents et des étudiants ont répondu à des questionnaires en ligne, composés de questions à choix multiples et à court développement, ce qui avait pour visée de faire une analyse approfondie des besoins des deux groupes susceptibles de participer à l'expérimentation d'OASIS+ (Milot et al., sous presse). Ces questionnaires ont permis de recueillir des informations auprès des parents et des étudiants au sujet : (1) de leur intérêt et de la façon

\footnotetext{
${ }^{3}$ Les acteurs qui forment le collectif de recherche impliqué dans le codéveloppement de ce programme sont cinq intervenants d'organismes communautaires de la région, deux parents, deux étudiants au $1^{\text {er }}$ cycle et deux professeurs de l'Université Laval. La création du programme, ainsi que son implantation, s'inscrivent dans une recherche évaluative participative sous le leadership des deux professeures.

${ }^{4}$ Ce projet de recherche a reçu l'approbation du comité d'éthique de la recherche de l'Institut de réadaptation physique de Québec (\#2017-547).
} 
dont ils souhaitaient s'impliquer; (2) de la formation des étudiants ; (3) des modalités de soutien désirées tout au long de leur implication; (4) des types de services à offrir aux familles, ainsi que (5) des facilitateurs susceptibles de favoriser leur engagement. Temps 3 . Des entrevues téléphoniques ont été effectuées avec 10 représentants d'organismes offrant des services de soutien à domicile ou dans la communauté à des familles vivant avec un enfant présentant une DI ou un TSA de tout âge et issues de différentes régions de la province de Québec. Le présent article porte sur les résultats de cette collecte de données, dont l'objectif était de comprendre le fonctionnement global de l'offre de services des organismes ciblés et d'identifier les obstacles et facilitateurs rencontrés lors de leur implantation.

\section{MÉTHODOLOGIE}

\section{Collecte de données}

\section{Recrutement des participants}

Pour identifier les facilitateurs liés à l'implantation d'une offre de services pérenne, des responsables de différents organismes de la province de Québec ont été invités à participer à des entrevues téléphoniques individuelles. Ces organismes ont été identifiés à partir d'une liste de ressources répertoriées par une association québécoise de familles vivant avec un enfant handicapé et de recherches sur Internet. Pour figurer sur cette liste, chaque organisme devait répondre au principal critère d'inclusion qui était d'offrir des services d'accompagnement, d'assistance ou de gardiennage à domicile ou dans la communauté ( $p$. ex. : dans les loisirs) à des familles dont un membre présente une DI ou un TSA. Cette liste de 15 organismes a été par la suite validée par les deux chercheures principales (EM et MG). Puis, un assistant de recherche a établi un contact par courriel ou par téléphone avec un responsable de chacun de ces organismes pour sonder l'intérêt à participer à la recherche et s'assurer que chaque organisme répondait au critère d'inclusion. Cet assistant a ensuite fixé un rendezvous pour une entrevue téléphonique d'environ 60 minutes avec les représentants des organismes qui

avaient manifesté leur intérêt. À ce moment, deux organismes supplémentaires ont été proposés par les répondants. Au final, les représentants de 10 organismes sur les 17 ciblés ont accepté de réaliser une entrevue.

\section{Outils de collecte de données}

Le guide d'entrevue semi-dirigée a été développé par l'équipe de recherche à la suite d'un atelier participatif avec le collectif de recherche. II a été créé dans l'intention de bien comprendre le fonctionnement global de l'offre de services des organismes ciblés et d'identifier les obstacles et facilitateurs rencontrés lors de leur implantation. Cette phase de la recherche visait aussi à dégager l'ensemble des conditions nécessaires au développement d'une offre de services durable. Les 14 questions du guide étaient regroupées en 5 sections. D'abord, les participants étaient invités à faire une description détaillée de l'offre de services développée par leur organisme en présentant les critères à respecter pour en bénéficier, les frais qui y étaient associés et les aspects les plus appréciés des familles. Ensuite, ils ont décrit les moyens utilisés pour rejoindre ces dernières. Puis, les participants se sont prononcés sur les actions entreprises pour favoriser le recrutement de personnes-ressources compétentes afin de donner des services variés et continus aux familles. Les organismes offrant de la formation spécifique préalable à l'implication auprès des familles étaient aussi invités à développer ce 
sujet. Ensuite, les informateurs ont partagé leurs idées sur les conditions favorables à une coordination efficace de l'offre de services, tout en mettant en lumière les facilitateurs et les obstacles qu'ils ont rencontrés. Finalement, ils ont exposé les démarches qu'ils ont faites et celles qu'ils auraient souhaité faire pour assurer la pérennité de leur offre de services, en abordant également la question du financement. La majorité des entrevues ont été réalisées par téléphone à l'exception d'une situation où l'entretien a été fait dans les locaux de l'organisme, à la demande du représentant. Toutes les entrevues ont été enregistrées et les propos ont été retranscrits intégralement.

\section{Analyse des données}

Les propos issus des entrevues ont fait l'objet d'une analyse de contenu thématique, au moyen d'une approche mixte, soit à la fois déductive et inductive (Miles et Huberman, 2003). Une première codification a été effectuée par l'assistant de recherche à partir des catégories élaborées dans le guide d'entrevue, laquelle a été validée par les deux chercheures principales. Par la suite, une démarche inductive a permis d'étayer et d'enrichir le processus d'analyse, ce qui s'est fait en utilisant le logiciel NVivo 11. Pour assurer la validité de la démarche, une professionnelle de recherche et une chercheuse ont validé l'arborescence de codes en considérant $20 \%$ du matériel codé.

\section{RÉSULTATS}

\section{Description de l'échantillon}

Parmi les 53 organismes répertoriés, 15 répondaient au principal critère d'inclusion. Un nombre important d'organismes ont été exclus, car leurs services étaient principalement offerts en maison de répit, à l'extérieur du domicile et des espaces de la vie communautaire. Les 10 organismes retenus se situent dans sept régions du Québec, soit : Montréal $(n=3)$; Montérégie $(n=2)$; Lanaudière $(n=1)$, Capitale-Nationale $(n=1)$, Chaudière-Appalaches $(n=1)$, Mauricie $(n=1)$ et Saguenay-Lac-SaintJean $(n=1)$. Parmi ceux-ci, trois offrent des services mixtes (au domicile et dans la communauté), six concentrent leur offre uniquement au domicile des familles et un seul offre ses services dans la communauté.

\section{Éléments à considérer pour l'implantation d'une offre de services pérenne}

Les points de vue des représentants concernant les leçons qu'ils ont tirées de l'implantation d'une offre de services pérenne sont abordés dans cette section. Les données qualitatives recueillies se rapportent à quatre questionnements, soit : (1) comment rejoindre les familles ? ; (2) comment s'assurer d'avoir des ressources compétentes pour offrir différents soutiens aux familles ? ; (3) comment favoriser une coordination efficace du service ? ; (4) comment assurer le financement du service ?

\section{Comment rejoindre les familles ?}

Pour faire connaître l'offre de services lors de sa création, les responsables d'organismes ont utilisé des façons de faire variées, soit en établissant des contacts privilégiés avec des professionnels du réseau de la santé et des services sociaux $(n=2)$, en effectuant la diffusion de publicités dans les établissements scolaires, dans les journaux locaux ou dans les lieux fréquentés par les familles (centres de la petite enfance, cliniques médicales, hôpitaux) $(n=2)$, ou encore en créant une page Facebook ou 
un site sur Internet $(n=2)$ pour faire connaître leurs services sur les réseaux sociaux. Néanmoins, la principale stratégie utilisée par chaque organisme a été de faire de la publicité auprès des professionnels du réseau de la santé et des services sociaux issus des $\mathrm{Cl}(\mathrm{U})$ SSS qui interviennent directement auprès des familles. Or, tous ont souligné avoir cessé d'utiliser cette méthode en raison, d'une part, du nombre croissant de familles intéressées par leur offre de soutien et, d'autre part, de leur difficulté à répondre à cette forte demande par manque de personnel. À ce sujet, un participant a dit avoir observé une augmentation de $27 \%$ des demandes des familles en une année. Somme toute, tous ont rapporté que les références de professionnels du réseau de la santé et des services sociaux, les partenariats établis avec d'autres organismes communautaires et le bouche-à-oreille étaient suffisants pour rejoindre les familles intéressées à bénéficier de l'offre de services.

Comment s'assurer d'avoir des ressources compétentes pour offrir des services variés et continus aux familles?

Selon les répondants, plusieurs actions nécessaires pour offrir des services de qualité, personnalisés et adaptés aux besoins évolutifs des familles. Dans ce but, le recrutement de personnes-ressources compétentes et fiables représente un élément crucial. L'extrait suivant fait ressortir cet aspect :

Le premier défi, c'est les ressources humaines. C'est d'embaucher les bonnes personnes. Ça, c'est un défi parce que si ça ne marche pas, si on se rend compte, au bout de trois mois, que ça ne marche pas, il faut recommencer, puis il faut recommencer à faire des entrevues de sélection. Puis [...] Bien, il faut reformer. On perd beaucoup de temps. (S8)

Cet extrait laisse entrevoir que l'embauche de personnes-ressources qui détiennent les compétences nécessaires exige un investissement de temps substantiel. À ce sujet, la moitié des participants $(n=5)$ ont rapporté faire des entrevues de sélection avec les candidats qui soumettent leur candidature à l'organisme. Plusieurs répondants $(n=6)$ ont aussi mentionné que dès l'embauche, ils soutiennent la préparation des personnes-ressources qui offriront des services aux familles, par de la formation ou des rencontres individuelles. Ils s'assurent aussi que ces nouvelles recrues comprennent bien la mission de l'organisme, de même que les tâches qu'elles devront réaliser auprès des familles. Les enjeux relatifs aux déplacements sont aussi abordés lors de l'embauche du personnel. En effet, certains organismes couvrent un vaste territoire, et les personnes-ressources concernées doivent planifier leurs déplacements pour offrir les services aux familles. Certains informateurs $(n=2)$ ont également noté l'importance de s'assurer que les personnes-ressources s'approprient bien leur rôle et que les frontières de celui-ci leur apparaissent bien claires. Entre autres, ces personnes ne doivent jamais réaliser des interventions thérapeutiques, mais plutôt offrir différents services de gardiennage ou d'accompagnement, selon les besoins des familles.

Pour soutenir les personnes-ressources à court et à moyen termes, différentes stratégies sont adoptées par les représentants d'organismes, telles que des suivis téléphoniques ou en personne et des rencontres individuelles mensuelles. La majorité des informateurs ont mentionné faire un suivi auprès des nouvelles personnes-ressources après la première rencontre avec une famille. Les familles peuvent également communiquer en tout temps avec les responsables des organismes, au besoin.

Des répondants ont confié que pour favoriser la continuité des services offerts aux familles, il est essentiel de favoriser des jumelages « personne-ressource/famille » qui respectent certains critères. À ce sujet, tous les répondants réalisent cette démarche en tenant compte de l'expertise, de la disponibilité 
et des intérêts des personnes-ressources. La majorité $(n=8)$ d'entre eux ont rapporté aussi se référer d'abord aux besoins et préférences des familles, avant d'effectuer tout jumelage. Plusieurs $(n=5)$ ont indiqué que la création du lien de confiance entre l'enfant et la personne-ressource ne doit pas être négligée. Lorsque le lien ne se développe pas entre la famille et la personne-ressource, ou qu'il y a de l'insatisfaction venant d'une part ou de l'autre, les représentants d'organismes doivent trouver une nouvelle personne-ressource pour offrir du soutien à la famille. Plusieurs répondants ont indiqué qu'il ne fallait pas forcer les choses lors d'une telle situation mais plutôt amorcer d'autres démarches pour créer un pairage qui réponde mieux aux besoins de l'enfant, de la famille et de la personne-ressource. C'est ce qu'aborde un informateur avec ces propos :

[Pour plusieurs parents], faire confiance, ce n'est pas facile. Ouvrir sa porte et tout ça... Alors, chez nous là, il faut que ça fonctionne. [...] Des fois, il y a des conflits de personnalités, puis on ne force pas les choses. II faut vraiment qu'on sente qu'il y a une affinité entre la famille [...]. Puis, les parents peuvent avoir une affinité avec [la personne-ressource], mais [avec] l'enfant ça ne fonctionne pas [...]. Bien, on recommence. On rebrasse les affaires, puis on offre les affaires autrement. (S7)

Les informateurs ont nommé plusieurs défis quant à l'accès et à la rétention du personnel, ce qui nuit à la continuité de l'offre de services aux familles. Ils ont souligné que lorsqu'une personne-ressource appréciée par une famille doit être remplacée pour différentes raisons (p. ex. : changement d'emploi), les parents ont souvent besoin d'un temps pour s'adapter. C'est ce que rapporte ce répondant : " Je vous dirais aussi que quand une [personne-ressource] est appréciée beaucoup dans une famille, quand elle quitte puis que tu fais un autre jumelage, on dirait que les attentes sont beaucoup plus élevées à ce moment-là parce qu'il y a des points de comparaison. Les familles font des points de comparaison, mais c'est le temps de s'adapter. »(S3)

En effet, cet extrait illustre bien le désir des familles d'accéder à une certaine continuité, ce qui n'est pas toujours facile. D'ailleurs, les répondants constatent que les familles sont souvent réticentes à faire part de leurs insatisfactions, par crainte de perdre le service et d'avoir à laisser entrer une nouvelle personne dans leur vie. Plusieurs répondants constatent aussi qu'un nombre plus restreint de personnes-ressources sont disponibles lors de moments où les familles en auraient davantage besoin, tel que lors du retour de l'enfant à la maison après l'école. De plus, peu de personnes-ressources acceptent d'offrir des services $24 \mathrm{~h} / 24$, ce qui est souhaité par quelques parents. À ce sujet, un représentant d'organisme tient le discours suivant :

L'idéal, ça serait d'avoir un gardien ou un accompagnateur temps plein qui serait à ma disposition puis là, les familles m'appellent puis : «OK, tu t'en vas là. Après ça, tu t'en vas là », pour répondre à leurs besoins, parce que des fois, les demandes [...] surtout les demandes qui sont après l'école, en semaine, je sais que c'est celles-là qui sont plus dures à combler parce que soit la famille n'a pas de service de garde et que l'enfant ne peut pas rester seul à la maison... Donc, ça, ce sont de grands défis. (S1)

Du point de vue des répondants, un des facteurs permettant d'expliquer le roulement élevé du personnel serait lié à l'éloignement géographique des familles, ce qui, à moyen et long terme, découragerait plusieurs personnes-ressources engagées auprès de ces familles, surtout lorsqu'elles s'y rendent pour des périodes de temps plus courtes. Pour contourner cet irritant, des organismes offrent de rembourser les dépenses liées aux déplacements, en tout ou en partie, ou de couvrir les frais de transport en commun pour se rendre au domicile des familles. 
Aucun organisme n'a précisé avoir été en mesure de créer une liste de remplaçants, étant donné leur nombre restreint d'employés et leur souci d'assurer une continuité en jumelant une seule personneressource par famille, lorsque possible. Certaines familles demeurent longtemps sur les listes d'attente en raison des besoins complexes de leur enfant et du manque d'employés ayant l'expertise nécessaire et l'intérêt relativement aux services demandés. À ce sujet, un représentant d'organisme a relevé qu'il n'était pas toujours facile de répondre à leur demande : "Ce n'est jamais garanti pour toutes les demandes. Autant ça peut aller super vite, le jour même je trouve quelqu'un de disponible puis intéressé [...] autant que là, je dois faire du nouveau recrutement. Ça peut prendre un peu plus de temps. Autant que parfois, malheureusement, je ne trouve pas pour la demande. »(S1)

Tous les représentants ont souligné qu'accéder à une liste de remplaçants pourrait leur permettre de coordonner le service plus efficacement. Plusieurs $(n=6)$ étaient d'avis que la création d'un plan de la relève permettrait de préparer le départ d'employés, de même que de partager les tâches de gestion plus équitablement entre les différents employés de l'organisme. Selon ces répondants, un tel plan permettrait d'assurer la pérennité de l'offre de services. Toutefois, la création d'une liste de remplaçants et d'un plan de la relève n'est pas simple, étant donné l'augmentation constante de la demande des familles année après année. De plus, ces répondants craignent le surcroît de travail que la création d'une liste de remplaçants leur apporterait puisqu'ils ont l'impression qu'ils seraient constamment amenés à former du nouveau personnel. Ainsi, de leur point de vue, le temps qu'il faudrait investir pour réaliser une telle tâche aurait un effet considérable sur leur capacité à effectuer des suivis prompts et de qualité auprès du personnel et des familles impliquées. En outre, la création d'une liste de remplaçants nécessiterait non seulement un recrutement constant de personnes-ressources, mais aussi l'apport de modifications fréquentes au plan d'action annuel. Cela nuirait également à une coordination efficace de l'offre de services.

\section{Comment favoriser une coordination efficace?}

Une coordination adéquate est aussi un élément essentiel au bon fonctionnement de l'offre de services aux familles. Pour tous les organismes, la gestion repose sur le travail d'un coordonnateur à temps plein dont les tâches sont d'effectuer le recrutement et le jumelage et de faire des suivis auprès des familles et des personnes-ressources. Quelques représentants d'organismes $(n=3)$ ont indiqué que ce coordonnateur doit détenir certaines aptitudes et qualités pour assurer le bon fonctionnement de l'offre de services et favoriser sa pérennité. Parmi celles-ci, ils évoquent des aptitudes telles que l'organisation et la gestion du temps, ainsi qu'une bonne capacité d'adaptation et de la persévérance. Pour faciliter le travail du coordonnateur, les participants insistent sur l'importance d'avoir des outils de gestion adaptés (p. ex. : outil de compilation des demandes des familles, outil pour gérer les suivis réalisés) et un espace de travail à l'abri du bruit et permettant des échanges confidentiels pour s'entretenir avec les parents au téléphone ou en personne.

Le travail de coordination doit aussi s'appuyer sur l'élaboration d'un bon plan d'affaires, ce qui peut favoriser l'établissement de contacts avec les bailleurs de fonds et, ainsi, assurer plus facilement le financement. Avoir un outil informatique permettant de compiler des statistiques pertinentes et précises sur l'organisme (p. ex. : nombre de demandes, nombre de personnes-ressources, nombre d'heures réalisées) et sur les services offerts par le personnel est aussi identifié comme aidant. Ultimement, celuici faciliterait le développement de demandes de subvention plus étoffées. Afin de coordonner efficacement l'offre de services, un représentant d'organisme a mis en place une base de données 
interactive en ligne, qui est aussi accessible aux parents. Ces derniers peuvent rechercher une personne-ressource selon leurs besoins précis (p. ex. : disponibilités, âge, sexe) et la contacter directement. Cet outil allégerait grandement la tâche du coordonnateur. L'existence d'un conseil d'administration constitué de parents bénévoles engagés et de représentants d'autres organismes possédant un regard différent sur l'offre de services a également été identifiée comme un facteur facilitant la bonne coordination des activités. Selon des répondants, l'engagement d'un tel conseil d'administration permettrait d'orienter l'offre de services pour mieux répondre aux besoins des familles qui en bénéficient.

\section{Comment financer l'offre de services ?}

Tous les répondants ont signalé que l'accès au financement constituait l'obstacle principal à la survie de leur offre de services à travers le temps. Les subventions allouées doivent couvrir les frais associés à l'embauche des employés impliqués indirectement auprès des familles (p. ex. : coordonnateur, directeur) et au loyer, entre autres. Les représentants d'organismes interrogés ont l'impression de livrer un «perpétuel combat » pour accéder à un financement suffisant. En effet, la survie de l'offre de services dépend grandement des subventions dont les organismes bénéficient. Dans la majorité des cas, ils ont accès à des fonds de démarrage pour débuter leurs activités, d'une durée de deux à trois ans, puis ils doivent soumettre des demandes de subvention annuellement. Ainsi, les démarches à faire pour assurer la pérennité de l'offre doivent constamment être renouvelées, ce que relevait un participant tenant ces propos: “Le manque d'argent est toujours présent après trente ans. [...] sauf qu'il faut quand même être capable de survivre. »(S5)

Des répondants ont souligné que la plus-value d'une offre de services d'accompagnement, d'assistance ou de gardiennage adaptée aux besoins spécifiques de ces familles est difficile à faire reconnaître auprès des bailleurs de fonds et des instances gouvernementales concernées. Ainsi, quelques représentants d'organismes $(n=3)$ disaient avoir investi beaucoup d'efforts pour faire valoir la pertinence et l'utilité de leur offre de services. L'un d'eux songeait même à embaucher une personne à temps plein pour s'occuper du financement des activités de l'organisme. L'offre d'un salaire intéressant aux personnes-ressources impliquées directement auprès des familles représente un autre défi de taille puisque les budgets des organismes et des familles sont limités. Dans cinq organismes, les parents assument entièrement les frais en payant directement la personne-ressource. Dans trois organismes, les services sont gratuits et c'est l'organisme qui rémunère les personnes-ressources directement à partir d'une subvention. Dans un autre, les familles et l'organisme partagent à parts égales les frais associés au salaire de la personne-ressource. Dans un autre encore, c'est le CISSS de la région qui assume l'entièreté des dépenses entraînées par l'offre de services en fonction d'un nombre d'heures maximal établi pour chaque famille à la suite de l'évaluation de ses besoins. Aucun organisme n'a suggéré de miser sur l'engagement de personnes-ressources bénévoles pour offrir des services aux familles.

\section{Discussion}

Il faut préciser que les résultats présentés dans cet article visaient à identifier les facilitateurs liés à l'implantation d'une offre de services pérenne de répit et de soutien à la participation sociale destinée aux familles vivant avec un enfant présentant une DI ou un TSA, dans le but de guider le développement d'OASIS+. Ces résultats ont permis d'orienter les choix de l'équipe de recherche à travers l'élaboration 
d'OASIS+, une offre de services à domicile et dans la communauté qui est destinée à ces familles et qui mise sur la formation et l'implication d'étudiants universitaires en ergothérapie et en travail social. Les données recueillies laissent entrevoir la présence d'éléments-clés à considérer et qui se rapportent au soutien du coordonnateur, à la formation à offrir aux personnes-ressources, aux aspects à considérer pour favoriser leur implication et à la gestion efficace de l'offre de services. Des rapprochements entre ces éléments et les points de vue des parents et étudiants du territoire de la ville de Québec qui ont répondu aux questionnaires dans le cadre de ce projet (Milot et al., sous presse) sont proposés dans cette section. Il y est aussi question des liens avec les écrits scientifiques et des limites de cette étude.

Tout d'abord, les représentants ont référé à l'importance du travail du coordonnateur dans la gestion de l'offre de services. Celui-ci doit exercer différents rôles pour assurer la pérennité de cette offre, la satisfaction des familles et la rétention du personnel. En particulier, le coordonnateur doit être en mesure d'offrir un suivi adapté aux familles en créant un climat d'ouverture et de confidentialité, être à l'écoute de leurs inquiétudes et favoriser la prévention des conflits émergeant de leurs interactions avec la personne-ressource impliquée dans leur vie. Par ailleurs, les répondants constatent que certains parents auraient plus de difficulté à exprimer leurs critiques, par peur de perdre les services offerts et d'avoir à accueillir une nouvelle personne dans leur quotidien. D'ailleurs, deux études indiquent que le manque de continuité et la perte d'un service, même si celui-ci n'est pas à la hauteur des attentes, sont des motifs de préoccupation et de déception pour plusieurs parents (CFE, 2008 ; Resch et al., 2010). II faut préciser que ces parents sont souvent confrontés au bris du lien qui se crée avec les intervenants impliqués dans leur vie en raison du taux de roulement élevé au sein des établissements des $\mathrm{Cl}(\mathrm{U}) \mathrm{SSS}$. De ce fait, les parents ont souvent à accueillir dans leur vie familiale de nouveaux intervenants, à répéter leur histoire, à s'adapter au vocabulaire et à l'approche de chaque intervenant, ce qui peut mener à une certaine démobilisation (CFE, 2008). Pour prévenir, autant que possible, l'apparition d'une telle situation, le collectif de recherche entrevoit jumeler chaque famille à deux étudiants qui pourraient offrir des services en alternance (Milot et al., 2018). Ainsi, les services pourraient être fournis aux familles de manière continue, tout en respectant la réalité des étudiants participants.

Les résultats obtenus permettent de constater que le recrutement de personnes-ressources détenant les compétences nécessaires pour offrir des services de soutien et de répit de qualité à domicile et dans la communauté n'est pas sans défi. Habituellement, de telles ressources sont rares et les services sont habituellement onéreux et disponibles pour une période de temps limitée seulement (Protecteur du citoyen, 2009, 2012). Au Québec, certaines familles se tournent vers les ressources privées pour répondre à leurs besoins de soutien et de répit, ce qui peut représenter des frais importants (CFE, 2008) et contribuer aux inégalités entre les familles ayant des revenus plus élevés et celles dont la situation financière est plus précaire. II importe de mentionner également que même les ressources privées sont en nombre insuffisant au Québec et que leur personnel est souvent peu informé des spécificités de la DI et du TSA (CFE, 2008 ; Gauthier-Boudreault, Gallagher et Couture, 2017). Dans ce contexte, le développement d'une formation adaptée et d'un suivi régulier des acquis apparaît nécessaire. Or, cette recommandation peut être plus facile à implanter pour OASIS+, soit en contexte universitaire auprès de futurs intervenants intéressés à développer des compétences pertinentes à l'exercice de leur profession. À ce sujet, le collectif de recherche a estimé pertinent de mettre à contribution l'expertise des partenaires communautaires impliqués pour créer une formation portant sur les trois thèmes identifiés par les étudiants et les familles ayant participé aux questionnaires (caractéristiques associées à la DI et au TSA, gestion des comportements et partenariat avec les familles). Cette formation de type hybride comportera un volet en ligne, visant l'acquisition de savoirs, et un volet de formation en 
présence, pour mettre en application les nouvelles connaissances, ce qui a été suggéré par les étudiants ayant répondu au questionnaire (Milot et al., sous presse). Le collectif souhaite aussi soutenir la participation des étudiants issus de disciplines différentes à des ateliers de codéveloppement et d'analyse des pratiques (ACODAP) tout au long de leur implication (après 2, 5 et 7 mois de l'implantation qui durera 9 mois). Ces ateliers leur permettront: (a) d'échanger sur les situations vécues par les familles et sur les facteurs influençant leurs possibilités de participation sociale et (b) de réfléchir collectivement à des stratégies favorables à la création d'une meilleure alliance avec les familles. Outre la formation, d'autres facteurs sont à considérer pour favoriser la mise en place de conditions de travail intéressantes pour les personnes-ressources engagées auprès des familles. Les propos des représentants d'organismes laissent entrevoir que la proximité géographique et l'accès à un salaire intéressant sont des facilitateurs importants, ce qui a aussi été soulevé par les étudiants ayant répondu au questionnaire dans le cadre de cette étude (Milot et al., sous presse).

Les représentants ont aussi insisté sur l'importance de réaliser des démarches de financement continues. Ces démarches nous apparaissent nécessaires, et ce, non seulement pour s'assurer la pérennité de l'offre de services, mais aussi pour favoriser l'accès à ces services des familles à plus faibles revenus. À ce sujet, des efforts importants seront faits lors de l'implantation d'OASIS+ pour s'assurer que les frais encourus pour bénéficier des services soient abordables et adaptés à la capacité de payer des parents (p. ex. au travers des allocations dont ils bénéficient). De plus, une attention particulière sera portée au rapport coût/bénéfice afin qu'OASIS+ soit en mesure de solliciter des fonds durables pour assurer la coordination et lui permettre d'offrir des compensations aux familles dans le besoin. À cette fin, les fonds investis lors de l'implantation d'OASIS+ seront enregistrés séparément des dépenses encourues pour la recherche. À la suite de l'expérimentation, un soutien sera offert aux représentants des organismes communautaires participant au collectif de recherche qui assureront la gestion d'OASIS+ après l'expérimentation pour que son déploiement dans la région soit pérenne.

Finalement, il faut mentionner que les résultats présentés doivent être interprétés avec certaines précautions. Tout d'abord, l'équipe de recherche a sollicité uniquement des représentants d'organismes de la province de Québec, alors qu'il est fort possible que des programmes innovants et fort inspirants et dont la nature se rapprocherait davantage d'OASIS+ aient été développés au Canada ou à l'international. II est également possible que de nouvelles initiatives aient été développées au Québec et que l'équipe de recherche n'ait pas été en mesure de les identifier par la méthode utilisée. Par ailleurs, notons que le taux de participation des représentants dont l'organisme répondait au critère d'inclusion est fort intéressant (10/15), et qu'une certaine saturation a été atteinte au regard des données colligées, aspects qui renforcent la validité de la démarche scientifique déployée. Les résultats de cette démarche permettent d'identifier plusieurs dimensions à considérer dans toute offre de services à domicile et dans la communauté destinée aux familles dont un membre présente des incapacités.

\section{Conclusion}

Cet article met en lumière plusieurs éléments favorables au développement d'une offre de services de soutien pérenne pour les familles vivant avec un enfant qui présente une DI ou un TSA, éléments qui ont été identifiés en accédant à l'expérience de représentants d'organismes de la province de Québec. Ces éléments, combinés aux résultats de questionnaires remplis par des familles et des étudiants (Milot et al., sous presse), ont permis d'orienter les décisions du collectif de recherche impliqué dans le développement d'OASIS+, un programme misant sur la formation et l'engagement d'étudiants 
universitaires. La formation des étudiants, ainsi que le processus d'implantation et d'évaluation des effets d'OASIS+, sont présentement en préparation. Cette expérimentation permettra de recueillir des données pertinentes pour bonifier OASIS+ et favoriser le transfert vers d'autres contextes (en sollicitant aussi des étudiants d'autres disciplines et d'autres milieux de formation et en rejoignant aussi les familles avec un enfant présentant une déficience physique, à titre d'exemple). Somme toute, miser sur l'engagement d'étudiants apparaît une stratégie pertinente pour favoriser l'inclusion des familles et leur participation à la vie de leur communauté, tout en contribuant à la formation de futurs professionnels plus ouverts, curieux, empathiques et engagés.

\section{REMERCIEMENTS}

L'équipe de recherche souhaite remercier chaleureusement les membres du collectif de recherche pour leur précieuse implication ainsi que la Fondation Tanguay, le Centre interdisciplinaire de recherche en réadaptation et intégration sociale et le Consortium national de recherche sur l'intégration sociale pour leur soutien financier. 


\section{RÉFÉRENCES}

Agence de la santé et des services sociaux de la Capitale-Nationale (2009a). Cadre de gestionProgramme Soutien aux proches-aidants. Gardiennage, répit et dépannage. À l'intention de la clientèle ayant une déficience intellectuelle, une déficience physique et un trouble envahissant du développement, Québec, ASSS de la Capitale-Nationale.

Agence de la santé et des services sociaux de la Capitale-Nationale (2009b). Guide d'application Programme Soutien aux proches-aidants. Gardiennage, répit et dépannage. À l'intention de la clientèle ayant une déficience intellectuelle, une déficience physique ou un trouble envahissant du développement, Québec, ASSS de la Capitale-Nationale.

American Psychiatric Association (2013). Diagnostic and Statistical Manual of Mental Disorders DSM5 (5 éd.), Arlington, VA, American Psychiatric Publishing.

Bindels-De Heus, K. G., A. Van Staa, I. Van Vliet, F. V. Ewals et S. R. Hilberink (2013). «Transferring young people with profound intellectual and multiple disabilities from pediatric to adult medical care: Parents' experiences and recommendations ", Intellectual and Developmental Disabilities, vol. 51, $n^{\circ} 3$, p. $176-189$.

Brannan, A. M. et C. A. Heflinger (2006). "Caregiver, child, family, and service system contributors to caregiver strain in two child mental health service systems ", Journal of Behavioral Health Services \& Research, vol. 33, nº 4, p. 408-422.

Brisson, A., M. Bolduc et C. Jourdan-lonescu (2014). Étude descriptive de l'évaluation de comorbidité psychiatrique en déficience intellectuelle adulte, Revue francophone de la déficience intellectuelle, vol. 25 , p. $5-22$

Camirand, J., L. Dugas, J. F. Cardin, G. Dubé, V. Dumitru et C. Fournier (2010). Vivre avec une incapacité au Québec. Un portrait statistique à partir de l'Enquête sur la participation et les limitations d'activités de 2001 à 2006, Québec, Institut de la statistique de Québec.

Cappe, É., N. Poirier, C. Sankey, A. Belzil et C. Dionne (2018). « Quality of life of French Canadian parents raising a child with autism spectrum disorder and effects of psychosocial factors ", Quality of Life Research, vol. 27, n 4, p. 955-967.

Conseil de la famille et de l'enfance (CFE) (2008). Avis sur le soutien aux familles avec enfants handicapés. Trois priorités pour une meilleure qualité de vie, Québec, CFE.

Courcy, I. et C. Des Rivières-Pigeon (2013). "Déterminants sociaux de la santé et symptômes dépressifs chez les mères de jeunes enfants ayant un trouble du spectre de l'autisme (TSA) ", Canadian Journal of Community Mental Health/Revue canadienne de santé mentale communautaire, vol. 32, no 3 , p. $29-49$. 
Feldman, M., L. McDonald, L. Serbin, D. Stack, M. L. Secco et C. T. Yu (2007). "Predictors of depressive symptoms in primary caregivers of young children with or at risk for developmental delay ", Journal of Intellectual Disability Research, vol. 51, n 8, p. 606-619.

Gauthier-Boudreault, C., F. Gallagher et M. Couture (2017). "Specific needs of families of young adults with profound intellectual disability during and after transition to adulthood: What are we missing? ", Research in Developmental Disabilities, $\mathrm{n}^{\circ}$ 66, p. 16-26.

Institut national de santé publique du Québec (INSPQ) (2017). Surveillance du trouble du spectre de l'autisme au Québec, Québec, QC, INSPQ. https://www.inspq.qc.ca/publications/2310

Jancarik, A.-S. (2010). Recherche documentaire sur les taux de prévalence et le processus d'évaluation des troubles du spectre de l'autisme (TSA), Longueuil, Agence de la santé et des services sociaux de la Montérégie. http://www.autisme.qc.ca/assets/files/02-autisme-tsa/Recherche/2010$10 \_$Pre \%CC \%81valence \%20et \%20e \%CC \%81valuations \%20TSA.pdf

Ludlow, A., C. Skelly et P. Rohleder (2012). "Challenges faced by parents of children diagnosed with

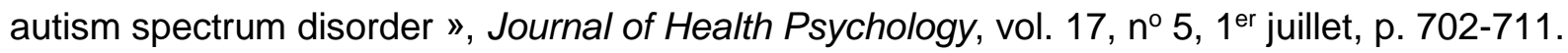

Luther, E. H., D. L. Canham et V. Young Cureton (2005). « Coping and social support for parents of children with autism », The Journal of School Nursing, vol. 21, n 1, p. 40.

Miles, M. B. et A. M. Huberman (2003). Analyse des données qualitatives, Paris, De Boeck Supérieur.

Milot, É., M. Grandisson, A.-S. Allaire, M. Couture, S. Tétreault, M. Chrétien-Vincent, C. Bédard et R. Couvrette (sous presse, 2018). "Miser sur l'engagement étudiant pour répondre aux besoins d'accompagnement des familles avec un enfant présentant une DI ou un TSA : points de vue d'étudiants et de parents ", Canadian Social Work Review.

Milot, É., M. Grandisson, S. Moisan, A. S. Allaire et C. Bédard (2018). Le programme OASIS DI-TSA. Mieux soutenir les familles tout en misant sur l'implication étudiante, Coll. "Soirée de la recherche de la Semaine québécoise de la déficience intellectuelle de l'Association pour l'intégration sociale (région de Québec) », Université Laval, Québec.

Munir, K. M., S. L., Friedman et L. S. Szymanski (2015). « Neurodevelopmental disorders: Intellectual disability ", dans A. Tasmanm, J. Kay, J. A. Lieberman, M. B. First et M. Maj, Psychiatry (4e éd.), Hoboken, NJ, John Wiley \& Sons, p. 672-705.

Murphy, N. A., B. Christian, D. A. Caplin et P. C. Young (2007). «The health of caregivers for children with disabilities: Caregiver perspectives ", Child Care and Health Development, vol. 33, n 2, p. 180 187.

Myers, B. J., V. H. Mackintosh et R. P. Goin-Kochel (2009). " "My greatest joy and my greatest heart ache": Parents' own words on how having a child in the autism spectrum has affected their lives and their families' lives ", Research in Autism Spectrum Disorders, vol. 3, n 3, p. 670-684. 
Office des personnes handicapées du Québec (2009). À part entière : pour un véritable exercice du droit à l'égalité. Politique gouvernementale pour accroître la participation sociale des personnes handicapées, Drummondville, OPHQ.

Ones, K., E. Yilmaz, B. Cetinkaya et N. Caglar (2005). « Assessment of the quality of life of mothers of children with cerebral palsy (primary caregivers) », Neurorehabilitation and Neural Repair, vol. 19, $\mathrm{n}^{\circ} 3$, p. 232.

Poirier, N. et C. Des Rivières-Pigeon (2013). Le trouble du spectre de l'autisme. État des connaissances, Québec, Presses de l'Université du Québec.

Protecteur du citoyen (2012). Rapport spécial du Protecteur du citoyen. Les services aux jeunes et aux adultes présentant un trouble envahissant du développement : de l'engagement gouvernemental à la réalité, Québec, Protecteur du citoyen.

Protecteur du citoyen (2009). Rapport spécial du Protecteur du citoyen sur les services gouvernementaux destinés aux enfants présentant un trouble envahissant du développement, Québec, Protecteur du citoyen.

Resch, J. A., G. Mireles, M. R. Benz, C. Grenwelge, R. Peterson et D. Zhang (2010). « Giving parents a voice: A qualitative study of the challenges experienced by parents of children with disabilities », Rehabilitation Psychology, vol. 55, n² 2, p. 139-150.

Siklos, S. et K. Kerns (2006). « Assessing need for social support in parents of children with autism and down syndrome », Journal of Autism and Developmental Disorders, vol. 36, n 7, p. 921-933.

Tétreault, S., P. Beaupré, N. Boucher, M. Carrière, H. Gascon, P. Marier-Deschênes et G. Piérart (2012a). Analyse des politiques sociales et des stratégies de soutien destinées aux familles vivant avec un enfant handicapé. Synthèse des connaissances sur les expériences actuelles ou récentes et propositions de solutions pour le Québec, Office québécois des personnes handicapées du Québec (OPHQ), Université Laval en collaboration avec le CIRRIS.

Tétreault, S., P. Beaupré, N. Boucher, M. Carrière, H. Gascon, P. Marier-Deschênes et G. Piérart (2012b). Recommandations de stratégies de soutien : pour assurer l'épanouissement des familles québécoises vivant avec un enfant handicapé (de la naissance à 17 ans), Québec, OPHQ.

Tétreault, S., S. Blais-Michaud, P. Marier-Deschênes, P. Beaupré, H. Gascon, N. Boucher et M. Carrière (2014). " How to support families of children with disabilities? An exploratory study of social support services », Child \& Family Social Work, vol. 19, n 3, p. 272-281.

Vézina, V., O. Bourdages et M.-H. Talbot (2015). Répit aux proches aidants (RAPA). Comprendre le processus d'évaluation des besoins. http://autismequebec.org/autisme_quebec_fichiers/file/Outil \%20RAPA \%20Doc.pdf 
Wallander, J. L., M. C. Dekker et H. M. Koot (2006). «Risk factors for psychopathology in children with intellectual disability: A prospective longitudinal population-based study », Journal of Intellectual Disability Research, vol.50, n 4, p. 259-268.

Woodgate, R. L., C. Ateah et L. Secco (2008). « Living in a world of our own: The experience of parents who have a child with autism », Qualitative Health Research, vol. 18, nº 8, $1^{\text {er }}$ août, p. 1075-1083. 